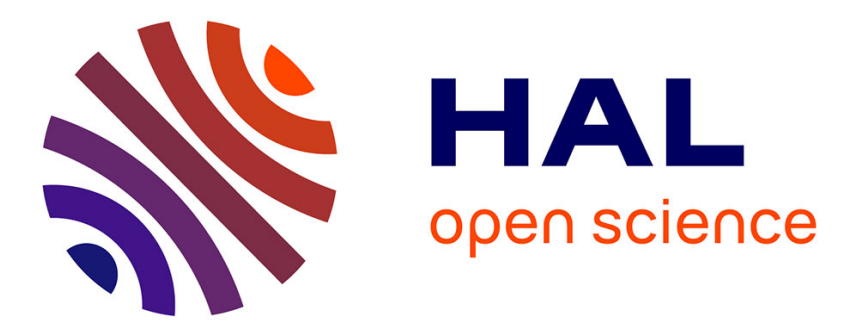

\title{
Measurement of laser absorptivity for operating parameters characteristic of laser drilling regime
}

\author{
Matthieu Schneider, Laurent Berthe, Rémy Fabbro, Maryse Muller
}

\section{To cite this version:}

Matthieu Schneider, Laurent Berthe, Rémy Fabbro, Maryse Muller. Measurement of laser absorptivity for operating parameters characteristic of laser drilling regime. Journal of Physics D: Applied Physics, 2008, 41 (15), pp.6. 10.1088/0022-3727/41/15/155502 . hal-00794960

\section{HAL Id: hal-00794960 \\ https://hal.science/hal-00794960}

Submitted on 28 Feb 2013

HAL is a multi-disciplinary open access archive for the deposit and dissemination of scientific research documents, whether they are published or not. The documents may come from teaching and research institutions in France or abroad, or from public or private research centers.
L'archive ouverte pluridisciplinaire HAL, est destinée au dépôt et à la diffusion de documents scientifiques de niveau recherche, publiés ou non, émanant des établissements d'enseignement et de recherche français ou étrangers, des laboratoires publics ou privés. 


\title{
Measurement of laser absorptivity for operating parameters characteristic of laser drilling regime
}

\author{
M Schneider, L Berthe, R Fabbro and M Muller
}

Laboratoire PIMM

Arts et Métiers Paristech

151 Boulevard de l'Hôpital 75013 Paris France

E-mail: matthieu.schneider@ensam.eu

\begin{abstract}
Laser drilling in the percussion regime is commonly used in the aircraft industry to drill sub-millimetre holes in metallic targets. Characteristic laser intensities in the range of $10 \mathrm{MW} \mathrm{cm}^{-2}$ are typically employed for drilling metallic targets. With these intensities the temperature of the irradiated matter is above the vaporization temperature and the drilling process is led by hydrodynamic effects. Although the main physical processes involved are identified, this process is not correctly understood or completely controlled. A major characteristic coefficient of laser-matter interaction for this regime, which is the absorptivity of the laser on the irradiated surface, is still unknown, because of the perturbing effects due to laser beam geometrical trapping inside the drilled hole. So, by using time resolved experiments, this study deals with the direct measurement of the variation of the intrinsic absorption of aluminium, nickel and steel materials, as a function of the incident laser intensity up to $20 \mathrm{MW} \mathrm{cm}{ }^{-2}$. We observe that for this incident intensity, the absorptivity can reach up to $80 \%$. This very high and unexpected value is discussed by considering the microscopic behaviour of the heated matter near the vapour-liquid interface that undergoes possible Rayleigh-Taylor instability or volume absorption.
\end{abstract}

\section{Introduction}

Laser drilling in the percussion regime is commonly used in the aircraft industry to drill sub-millimetre holes in metallic targets [1]. Laser intensity in the range of $10 \mathrm{MW} \mathrm{cm}^{-2}$ is typically used for drilling these metallic targets. At this level of intensity, the temperature of the irradiated matter is typically above the vaporization temperature and the drilling is led by efficient hydrodynamic effects. Previous authors have already discussed the mechanisms of the laser drilling [2]: the irradiated area heats, melts and gets evaporated. As a result of this strong surface vaporization, an induced pressure, called recoil pressure, is generated. Due to its 'piston effect', the recoil pressure applied onto this surface expels the melt part out of the hole. Thus the evaporation mechanism is the leading process of the laser drilling. Although the main physical processes involved are identified, this process is not totally understood and controlled because of the very rapid variation of thermodynamic parameters induced by the high incident laser intensity that occur on typical time scales of microseconds and scale lengths of micrometres inside these heated volumes.

Measurements of fundamental physical coefficients of laser-matter interaction are very difficult because of the complexity of the process and the resulting geometry. However, the determination of the absorption coefficient of the material for these high laser intensities is necessary: this issue is fundamental for a physical understanding of the process and more particularly useful as input for numerical simulations. Usually, the absorption coefficients given in the literature are for solid materials with totally smooth surfaces. But for very hot melted metals this measurement is difficult to realize 

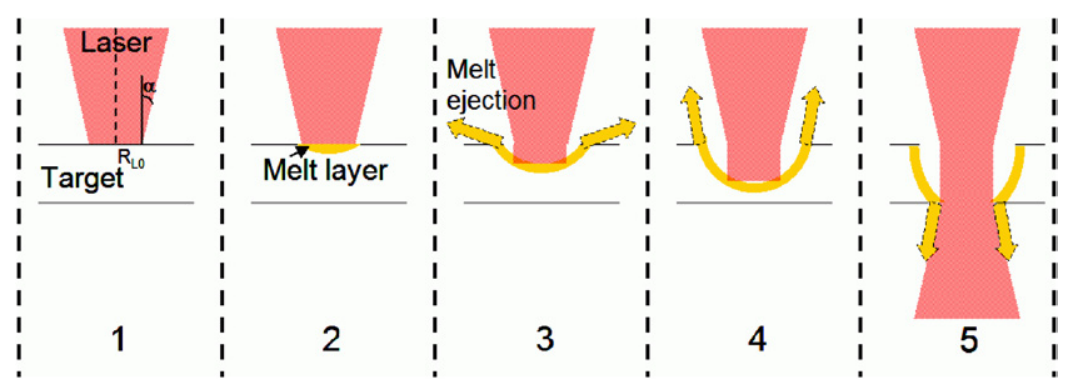

Figure 1. Schematic representation of laser drilling. $\mathrm{OR}_{\mathrm{L} 0}$ is the laser radius in the focal plan and $\alpha$ the numerical aperture. During drilling, the surface is heated (1), melted (2) and vaporized. The vapour flow becomes supersonic and the recoil pressure expels the melt layer out of the hole at the side of the focal spot, first horizontally (3) then vertically (4). At the end of the drilling, the hole breaks through (5).

because of the geometry of the sample and the nature of matter change above the melting temperature [3].

Several physical phenomena can increase the absorptivity. They result from the intrinsic properties of the material, as an effect of the temperature variation and the geometrical evolutions of the surface target. The main goal of this work is to estimate the absorptivity during drilling, by using thin targets in order to suppress the inherent geometrical trapping that occurs inside drilled holes with significant aspect ratios. Section 2 of this paper describes the physical processes involved in laser drilling in the percussion regime. The protocol and the experimental setup used are described in section 3. Section 4 presents and discusses the results obtained for a single pulse drilling and possible phenomena responsible for the observed variations. The reflectivity during drilling is given as a function of the laser peak power. The measurements show that this coefficient decreases to about $20 \%$ for the highest peak power we used, whatever the nature of material. Section 5 summarizes and concludes on each hypothesis discussed in the paper.

\section{Physical description of phenomena}

Laser drilling is the process of focusing the high-power laser beam onto a workpiece, melting and vaporizing the material to create a hole. In this work, the type of laser drilling is the percussion mode; the laser is static related to the workpiece during drilling. The whole hole is drilled along the optical axis. Laser drilling is sequenced in five steps, schematically represented on figure 1.

Firstly, the irradiated target surface is heated by absorption of laser energy (figure 1(1)). The absorptivity depends on three parameters: the material nature, the temperature and the surface state. The fraction of this absorptivity that is a function of the temperature and the material nature is called intrinsic absorptivity, and typically for steel it is about 0.35 at $1064 \mathrm{~nm}$ $[4,6]$. The surface roughness increases the absorptivity with multi-reflections occurring inside microscopic structures and the temperature is below the melting one.

Secondly, a thin layer on surface is melted (figure 1(2)), the surface becomes smooth and then the absorptivity is equal to the intrinsic one and is only a function of temperature. The temperature is then between the fusion and the vaporization one.
Table 1. Reflectivity of several elements at $1064 \mathrm{~nm}$ [5].

\begin{tabular}{llllllllllllll}
\hline & $\mathrm{Au}$ & $\mathrm{Ag}$ & $\mathrm{Cu}$ & $\mathrm{Al}$ & $\mathrm{Ta}$ & $\mathrm{Pt}$ & $\mathrm{Ni}$ & $\mathrm{Pd}$ & $\mathrm{Co}$ & $\mathrm{Fe}$ & $\mathrm{Cr}$ & $\mathrm{W}$ & $\mathrm{Ti}$ \\
\hline$R(\%)$ & 99 & 98 & 97 & 94 & 88 & 75 & 73 & 72 & 70 & 65 & 63 & 61 & 56 \\
\hline
\end{tabular}

Thirdly, the surface temperature is higher than the vaporization one. Then, the resulting saturated vapour pressure $\left(P_{\mathrm{s}}\right)$ is above the atmospheric one. It is known that the recoil pressure $\operatorname{Pr}$ is proportional to the saturated vapour; therefore, $\operatorname{Pr}=A P_{\mathrm{s}}$, with the factor $A$ being dependent on the ambient pressure and the saturated vapour pressure. Its value varies from 0.56 , for expansion into vacuum, to 1 , under high ambient pressure (see [2]). The vapour flow is ejected normally to the local surface. The recoil pressure generated by the evaporation exerts a force on the melted surface that expels the liquid layer out of the hole by the side (figure 1(3)), horizontally by the 'piston effect'.

Fourthly, the ejected vapour flow becomes supersonic, with a Mach shock disc (MSD) appearing in the flow [7]. The recoil pressure is equal to $0.56 P_{\mathrm{s}}$, and for these conditions, $P_{\mathrm{s}}$ is much higher than the atmospheric pressure. The irradiated surface propagates deeper inside the target. The ejection of the melt then becomes vertical (figure 1(4)).

Fifthly, the hole breaks through (figure 1(5)). The melt part is ejected through the rear face and the laser interaction stops.

Three phenomena might be cumulated during measurements of absorptivity on non-prepared surfaces.

The first one, called the intrinsic absorptivity, are the properties of the matter that depends on its optical index. Drude's model describing the electron behaviour inside the solid [4], combined with the laws of electromagnetism, leads to the determination of a complex optical index of material. Table 1 displays examples of intrinsic reflectivities $R$ of several elements at $1064 \mathrm{~nm}$ and $293 \mathrm{~K}$ temperature [5]. This coefficient depends on the wavelength, material nature and temperature. At $1064 \mathrm{~nm}$ wavelength and on the steel target, the absorptivity is between 0.3 and 0.4 for target temperatures less than or equal to the fusion temperature. When the metal becomes liquid, the absorptivity is modified and increases by about 5\% [6]. However, at the very beginning of the drilling in this regime, on a typical time scale of $50 \mu \mathrm{s}$ [7], the reached temperature easily exceeds the vaporization one and the variation of the intrinsic absorptivity is then unknown. 


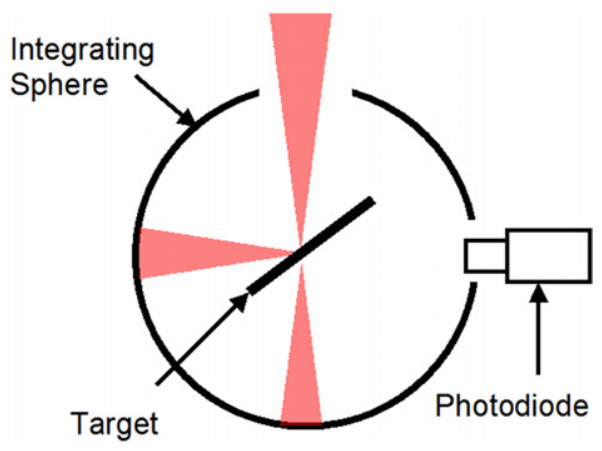

Figure 2. Experimental setup of the integrating sphere. A photodiode is used for recording the time evolution of the reflected laser beam. The target is tilted in order to collect the entire reflected laser beam.

The second phenomenon is laser energy absorption by the more or less ionized metal vapour. It is well known that when an electromagnetic wave propagates through a plasma, it can be reflected or absorbed in an inverse Bremsstrahlung process. Because of the rather low plume temperature, the absorption by the vapour plume above the target is negligible for the wavelength of $1064 \mathrm{~nm}$ and for the laser intensity used $\left(20 \mathrm{MW} \mathrm{cm}^{-2}\right)$ [8-10] and is discussed in this paper. The absorption area is then located near the surface of the target.

The third phenomenon is a geometrical evolution during laser drilling. It depends on the shape of the irradiated area underneath the focal spot. For a curved or a roughness surface some multiple reflections can occur and consequently perturb the measurement of the intrinsic absorption. The deformed surface by the recoil pressure can trap the laser beam. This consideration will be discussed later on.

So the interaction between laser and matter for these operating conditions is rather difficult to analyse because of the following.

- Matter is very hot, with the temperature above the vaporization one $\left(T_{\mathrm{vap}}=2770 \mathrm{~K}\right)$. The recoil pressure is higher than the atmospheric one [11-13].

- The vapour plume is emitted in a supersonic regime [7]. The recoil pressure modifies the liquid surface and thus the drilled hole morphology [14].

- The matter state is undetermined in the interaction area because of the high level of evaporated material flux [15].

\section{Experimental setup and analysis method}

Experimental setup. The experimental setup is shown in figure 2. It is based on a typical configuration for the study of absorption by using a calibrated integrating sphere. The YAG laser used is the HL201P from TRUMPF with a $1064 \mathrm{~nm}$ wavelength. The laser is focused onto the target surface and its spot diameter is $320 \mu \mathrm{m}$. The pulse duration is equal to $1 \mathrm{~ms}$. The whole laser parameters are frequently characterized. The results were published in $[7,16]$.

In order to recover the complete reflection, the target is tilted at an incidence angle of $30^{\circ}$. This tilt does not modify the intrinsic absorptivity of the metallic target [3], neither the

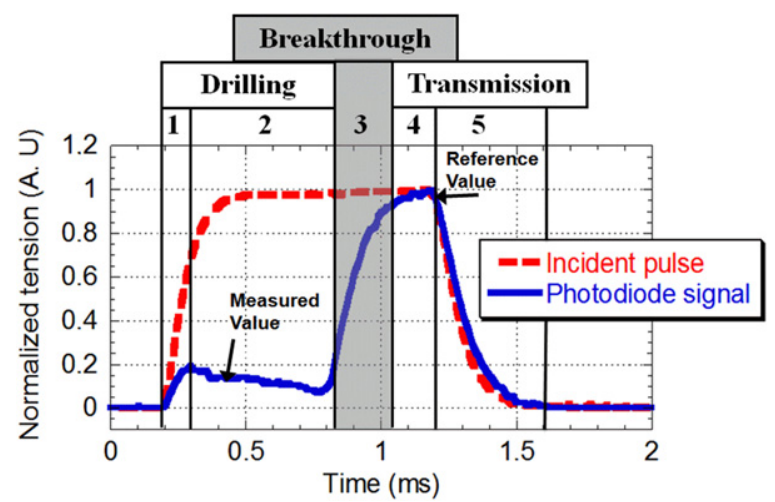

Figure 3. Typical signals recorder during drilling experiments. Incident pulse (dotted line) and reflected signal (continuous line).

involved physical phenomena [17]. The internal surface of the integrating sphere is covered with a $\mathrm{BaSO}_{4}$ layer whose reflectivity is very close to $100 \%$ at $1064 \mathrm{~nm}$. The photodiode is an FND 100 polarized in a linear regime with $12 \mathrm{~V}$ and has a typical response time of $10 \mathrm{~ns}$.

Three metallic materials have been studied: aluminium, nickel and steel. The target thickness is rather thin, typically $1 \mathrm{~mm}$, in order to suppress the perturbing effect of multiple reflections on the side walls of the drilled hole.

When using an integrating sphere, a preliminary calibration of the integrating sphere is generally necessary. However, for our specific drilling experiments of thin samples, this calibrating phase can be avoided, if one can consider that the transmitted laser pulse through the sample is characteristic of the incident pulse with no perturbation. Therefore, the target thickness should be thin enough to minimize the aspect ratio (depth/diameter) of the hole and to allow a total transmission through the hole. This point was of course confirmed by preliminary measurements. For the samples we used, the aspect ratio varied between 1.25 and 2 as a function of peak power [18].

Figure 3 shows the time dependence of the recorded signals given by the photodiode: The first one (dotted line) corresponds to the incident laser pulse, and the second one (continuous line) is the reflected one. The photodiode signal (continuous line) obtained during a drilling experiment is composed of five characteristic parts: parts 1 and 2 correspond to the reflected signal recorded by the photodiode during laser drilling before breakthrough. Part 3 corresponds to the hole breakthrough. Parts 4 and 5 is the transmission signal that corresponds to the incident signal where the reference value can be defined. The average coefficient of reflection is then defined by the ratio between the measured and the reference value:

$$
R\left(P_{\text {peak }}\right)=\frac{\text { measured value }}{\text { reference value }}
$$

\section{Results}

Figure 4 shows the evolution of reflectivity as a function of the incident peak power for three materials: aluminium (full dot), nickel (empty dot) and steel (square dot). Each point of the 


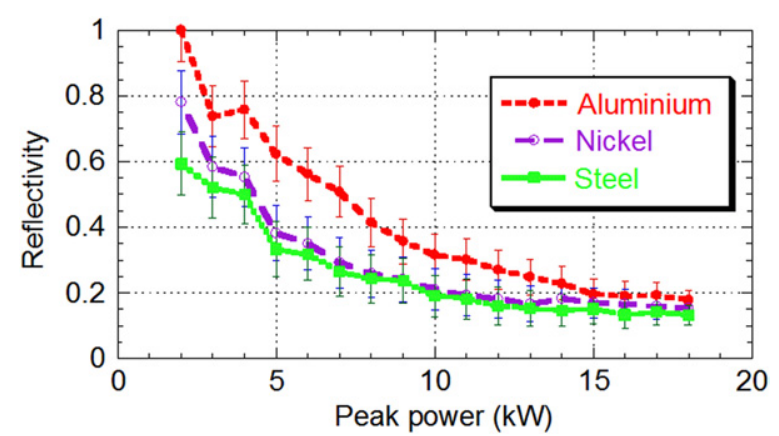

Figure 4. Reflectivity as a function of incident peak power, for three materials: aluminium (full dot), nickel (empty dot) and steel (square dot). Errors bars correspond to the reproducibility of 10 shots.

curves is obtained by the previous analysis method and the error bars result from the reproducibility of typically 10 shots.

The three curves present some fluctuations of reflectivity for the low peak powers, 3 and $4 \mathrm{~kW}$. These variations are explained by the modification of the matter state. the initial irradiated surface is rough; its reflectivity is lower or equal to the intrinsic reflectivity of a polished solid target to $1064 \mathrm{~nm}$. By increasing the peak power of the pulses, surface melts and becomes smooth like a polished surface. The coefficient of reflection increases and the measurement obtained gives the intrinsic value.

For $2 \mathrm{~kW}$ peak power (which corresponds to an incident intensity of $3 \mathrm{MW} \mathrm{cm}^{-2}$ ), the reflectivity measured is close to the intrinsic one at $1064 \mathrm{~nm}$ wavelength, considering the errors bars: 0.94 versus 1 for aluminium, 0.72 versus 0.78 for nickel and 0.65 versus 0.60 for steel.

For the highest peak power $(18 \mathrm{~kW}$, corresponding to $20 \mathrm{MW} \mathrm{cm}^{-2}$ ), the reflectivity decreases and reaches for all materials 0.13 for $\mathrm{Ni}, 0.15$ for steel and 0.18 for $\mathrm{Al}$ corresponding, respectively, to $87 \%, 85 \%$ and $82 \%$ of absorption.

\section{Discussion}

These reflectivities recorded for high peak power are very low and unexpected compared with the admitted values, which are generally between 0.4 and 0.6 in this regime. However, these experiments are reliable because they are confirmed:

- by the intrinsic reflectivity values obtained at a low peak power (typically less than $2 \mathrm{~kW}$ );

- by previous authors' measurements [19] obtained in the welding regime that would correspond to the medium peak power used here between 5 to $8 \mathrm{~kW}$.

Two reasons can explain these low values and are discussed in this section. As previously mentioned in the introduction, the absorption by the plasma plume is not relevant here.

The phenomenon of multiple reflections has to be considered at two different scales. The first one is on a macroscopic scale that corresponds to the hole size where the laser could make several reflections as a function of the hole profile and the aspect ratio. The second scale is in the range of the microscopic structure generated at the liquid/vapour interface.

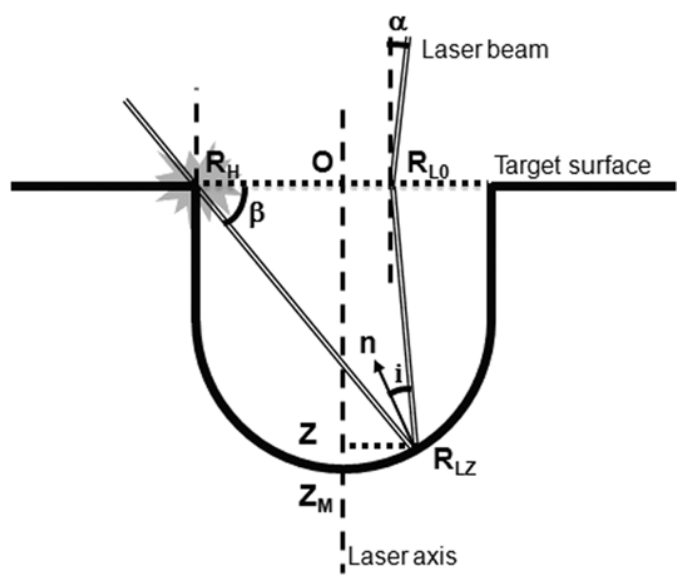

Figure 5. Scheme of a $U$ hole profile. $\mathrm{OR}_{\mathrm{H}}$ is the hole radius, $\mathrm{OR}_{\mathrm{L} 0}$ the laser radius in the focal plan, $\mathrm{OR}_{\mathrm{LZ}}$ its radius at the depth $\mathrm{z}, \alpha$ the numerical aperture, $\mathrm{n}$ is the normal at the local surface, $\mathrm{OZ}_{\mathrm{M}}$ is the maximal depth of the hole. OZ is the depth of laser periphery reflection. $i$ is the angle between the numerical aperture and the normal $n$. $\beta$ is the angle between the first reflected part of the beam that makes a second reflection in the hole with the surface.

Macroscopic scale and hole profile. The following approach estimates the hole depth for which the laser beam could undergo some multi-reflections. Figure 5 is a schematic representation of a hole drilled with a $U$ profile and a laser beam characterized by its numerical aperture $(\alpha)$ and its radius in the focal plane $\left(O R_{\mathrm{L} 0}\right)$. The hole bottom is considered hemispherical with a radius equal to the hole radius $\left(O R_{\mathrm{H}}\right)$. The external part of the beam is the first one to make possible several reflections inside the hole, as shown in figure 5. This multi-reflection will occur for a minimum depth $Z_{M}$ that we can estimate.

With the notation of figure 5, one can write the set of following equations:

$$
\begin{gathered}
\cos (\alpha)=\frac{O Z}{R_{L 0} R_{L Z}}, \\
\frac{R_{L 0} R_{H}}{\sin (2 i)}=\frac{R_{L 0} R_{L Z}}{\sin (\beta)}, \\
\sin (i+\alpha)=\frac{Z R_{L Z}}{O R_{H}}, \\
\sin (i+\beta)=1-\frac{Z Z_{M}}{O R_{H}},
\end{gathered}
$$

with $O^{\prime} R_{L Z}=O^{\prime} Z_{M}=O R_{H}$. Equations (1)-(3) lead to

$$
O Z=R_{L 0} R_{H} \cos (\alpha) \frac{\cos \left[\alpha-2 \times \arcsin \left(\frac{Z R_{L Z}}{O R_{H}}\right)\right]}{\sin \left\{2\left[\arcsin \left(\frac{Z R_{L Z}}{O R_{H}}\right)-\alpha\right]\right\}} .
$$

With a geometrical optics description of the laser radius evolution, equation (6) is easily found:

$$
Z R_{L Z}=O R_{L 0}+O Z \times \tan (\alpha) .
$$

Equation (4) gives

$$
Z Z_{M}=O R_{H}\left(1-\sqrt{1-\left(\frac{Z R_{L Z}}{O R_{H}}\right)^{2}}\right) .
$$




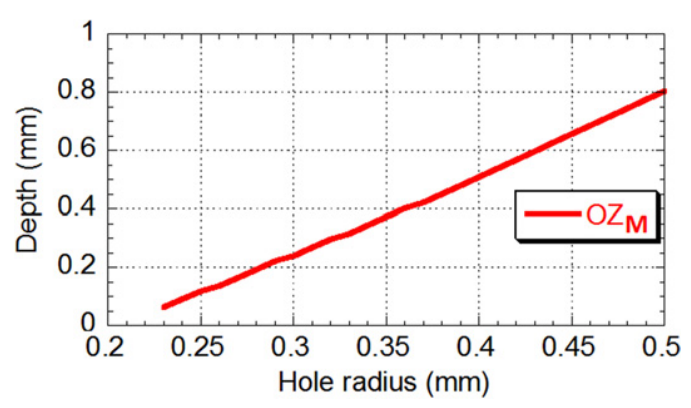

Figure 6. Depth at which multi-reflections occurs as a function of hole radius, for our operating conditions.

From figure 5, one can show that the beginning of multireflections will occur for a hole depth $\mathrm{OZ}_{\mathrm{M}}$ higher than

$$
\begin{aligned}
O Z_{M} & >R_{H} R_{L 0} \frac{\cos (\alpha) \cos \left[2 \times \arcsin \left(\frac{Z R_{L Z}}{O R_{H}}\right)-\alpha\right]}{\sin \left\{2\left[\arcsin \left(\frac{Z R_{L Z}}{O R_{H}}\right)-\alpha\right]\right\}} \\
& +O R_{H}\left(1-\sqrt{1-\left(\frac{Z R_{L Z}}{O R_{H}}\right)^{2}}\right)
\end{aligned}
$$

Figure 6 shows the evolution of $O Z_{\mathrm{M}}$ as a function of the hole radius. For hole radii between 0.35 and $0.5 \mathrm{~mm}$, multi-reflections appear when the depth reaches $0.4-0.8 \mathrm{~mm}$. As the drilling velocity is in the range of $2 \mathrm{~mm} \mathrm{~ms}^{-1}$ [14], the phenomenon of multi-reflection will appear $0.2 \mathrm{~ms}$ after the pulse beginning, corresponding to the measurement time (see figure 3). Therefore, one can be confident that our measurements are recorded for single laser reflection conditions inside the hole.

Moreover, as the target thickness is constant and the hole diameter increases with the peak power, the corresponding aspect ratio decreases. Then the geometric evolution of the hole reduces the probability of a multi-reflection phenomenon.

Microscopic scale: onset of Rayleigh-Taylor instability and volume absorption. Close to the liquid surface and because of the very high vapour flow, two microscopic phenomena can occur. The first one is the development of Rayleigh-Taylor instability. The second one is the growth of an absorbing layer from the surface. These two phenomena are located near or at the surface. They are a consequence of the irradiated matter behaviour for this high intensity and so they increase the intrinsic absorptivity.

It is well known that Rayleigh-Taylor instability occurs when a heavy fluid is accelerated by a light fluid. Under our conditions, the metallic vapour (light fluid) accelerates the liquid metal as a result of induced recoil pressure. It is also known that the non-linear stages of this instability leads to the development of geometric structures composed of the heavy fluid (here the liquid metal) penetrating inside the vapour that generates a mixing layer [20]. These structures, called Rayleigh-Taylor fingers, are characterized by high aspect ratios and therefore they could be good candidates for ensuring a local geometric trapping of an incoming laser beam. As a consequence of this induced roughness, the reflectivity of this surface should efficiently decrease.
The characteristic time $t_{\mathrm{m}}$ and the most unstable wavelength $\lambda_{\mathrm{m}}$ for the development of the mixing layer during the Rayleigh-Taylor instability are given by [20]

$$
t_{\mathrm{m}}=\left(\frac{\lambda_{\mathrm{m}}}{\pi G \alpha}\right)^{1 / 2} \quad \text { where } \lambda_{\mathrm{m}}=4 \pi\left(\frac{v^{2} \alpha}{G}\right)^{1 / 3},
$$

where for our conditions $\alpha$ is the Atwood number close to 1 , $v$ the kinetic viscosity is about $10^{-7} \mathrm{~m} \mathrm{~s}^{-2}$ for metallic liquid and $G$ is the acceleration field given by the ratio of the drilling velocity $V_{\mathrm{d}}$ and the pulse duration $\tau$, typically $2 \times 10^{3} \mathrm{~m} \mathrm{~s}^{-2}$. One then obtains $t_{\mathrm{m}}=60 \mu \mathrm{s}$ and $\lambda_{\mathrm{m}}=20 \mu \mathrm{m}$; as $t_{\mathrm{m}}$ is shorter than our measurement time (which is about $200 \mu \mathrm{s}$, see figure 3), one can expect that this instability has time to reach its non-linear phase.

For these intense laser irradiations, the evaporation process is very important. For these conditions of evaporation, the physical state of material near the liquid/vapour interface is very complex and quite undetermined: matter is in a high nonequilibrium state [21] with a probably very high gradient of its properties evolving from the dense phase to the supersaturated state [22] inside which micro- or nano-droplets induce Mie scattering of the incoming beam. Thus, volume absorption by this process may also play an important role in these conditions.

\section{Conclusion}

In this paper the intrinsic absorptivity during the laser drilling process has been measured as a function of the incident peak power (and thus incident intensity) on three different materials: aluminium, nickel and steel. We have used an integrating sphere that allowed us to obtain time resolved measurements and therefore to avoid their perturbation induced by the geometrical trapping of the laser beam inside the drilled hole. For laser intensity regimes corresponding to the onset of melting or to the laser welding process, the reflectivity measurements obtained (typically ranging from 50\% to 70\%) were in agreement with previous experiments. For higher laser intensities, a characteristic of the laser drilling process in the range $10-20 \mathrm{MW} \mathrm{cm}^{-2}$, the absorptivity is very high and typically reaches $80 \%$ whatever the nature of material used.

In order to explain such high absorptivity coefficients that are independent of the material used, we can speculate that several possible mechanisms induce strong perturbation on a microscopic scale at the liquid/vapour interface: RayleighTaylor instability probably increases the roughness of this interface, and also strong local evaporation probably favours scattering and absorption inside the disperse medium at this interface.

\section{References}

[1] McNally C A, Folkes J Pashby I R 2004 Laser drilling of cooling holes in aeroengines: state of the art and future challenges Mater. Sci. Technol. 20 805-13

[2] Semak V V and Matsunawa A 1997 The role of recoil pressure in energy balance during laser materials processing J. Phys. D: Appl. Phys. 30 2541-52 
[3] Samokhin A A 1990 First order phase transitions induced by laser radiation in absorbing condensed matter Effect of Laser Radiation on Absorbing Condensed Matter vol 13, pp 1-47 (Prokhorov edition)

[4] Ashcroft N W and Mermin N D 1976 Physique des Solides (EDP Sciences)

[5] Weast R C 1985 CRC Handbook of Chemistry and Physics 65th edition (Cleveland, OH: CRC)

[6] Dausinger F and Shen J 1993 Energy coupling efficiency in laser surface treatment ISIJ Int. 33 925-33

[7] Schneider M, Fabbro R, Berthe L and Muller M 2007 Gas investigation on laser drilling J. Laser Appl. 19165

[8] Mahrle A and Beyer E 2006 Hybrid laser beam welding-classification, characteristics, and applications J. Laser Appl. 18 169-80

[9] Delcroix J L and Bers A 1994 Physique des Plasmas (CNRS Editions, EDP Sciences)

[10] Kawahito Y, Kinoshita K, Katayama S, Tsubota S and Ishide T 2005 Visualization of interaction between laser beam and YAG-induced plume Proc. ICALEO'05 (Miami, USA) pp 920-8

[11] Kuznetsov L I 1994 Recoil momentum at a solid surface during developed laser ablation Quantum Electron 23 1035-8

[12] Landau L D and Lifshitz E M 1989 Physique Statistique 2nd edn

[13] Chen X and Wang H X 2001 A calculation model for the evaporation recoil pressure in laser material processing J. Phys. D: Appl. Phys. 34 2637-42
[14] Schneider M, Fabbro R, Berthe L and Muller M 2007 Study of hole properties in percussion regime with a new analysis method J. Laser Micro/Nanoeng. 2 128-32

[15] Knight C J 1979 Theorical modelling of rapid surface vaporization with back pressure AAIA J. 17 519-23

[16] Schneider M, Fabbro R, Berthe L, Landai L, Nivard M and Laurens P 2004 Parametric study of drilling with new innovative laser source: application to percussion regime Proc. ICALEO'04 (San Francisco, CA) pp 540-6

[17] Schneider M, Fabbro R, Berthe L and Muller M 2007, Influence of incident angle on laser drilling Proc. ICALEO'07 (Orlando, FL)

[18] Schneider M, Fabbro R, Berthe L and Muller M 2006 New experimental approach to study laser matter interaction during drilling in percussion regime $J$. Laser Micro/Nanoeng. 2 117-22

[19] Fabbro R, Slimani S, Coste F and Briand F 2005 Study of keyhole behaviour for full penetration NdYAG CW laser welding J. Phys. D: Appl. Phys. 38 1881-7

[20] Youngs D L 1984 Numerical simulation of turbulent mixing by Rayleigh-Taylor Instability Physica D 1232

[21] Finke B R, Finke M, Kapadia P D, Dowden J M and Simon G 1990 Numerical investigation of the Knudsen-layer, appearing in the laser-induced evaporation of metal Proc. SPIE 1279 127-34 (Laser Assisted Processing II)

[22] Labuntsov D A and Kryukov A P 1979 Analysis of intensive evaporation and condensation J. Heat Mass Transfer 22 989-1002 